\title{
Circ-ANAPC7 is Upregulated in Acute Myeloid Leukemia and Appears to Target the MiR-181 Family
}

\author{
Hongli Chen ${ }^{\mathrm{a}}$ Tian Liu ${ }^{\mathrm{a}}$ Jing Liu ${ }^{\mathrm{a}}$ Yuandong Fenga Baiyan Wang ${ }^{\mathrm{a}}$ Jianli Wang ${ }^{\mathrm{a}}$ \\ Ju Bai ${ }^{a}$ Wanhong Zhao ${ }^{a}$ Ying Shen ${ }^{a}$ Xiaman Wang ${ }^{a}$ Juan Yang ${ }^{b}$ Yuqiang Jic \\ Aili He $\mathrm{H}^{\mathrm{a}, \mathrm{d}}$ Yun Yang ${ }^{\mathrm{a}}$ \\ aDepartment of Hematology, The Second Affiliated Hospital of Xi'an Jiaotong University, Xi'an, \\ 'Department of Cell Biology and Genetics, School of Basic Medical Sciences, Xi'an Jiaotong University \\ Health Science Center, Xi'an, 'Department of Central Laboratory, The First Hospital of Xi'an City, Xi'an, \\ ${ }^{d}$ National-Local Joint Engineering Research Center of Biodiagnostics and Biotherapy, The Second \\ Affiliated Hospital of Xi'an Jiaotong University, Xi'an, China
}

\section{Key Words}

Acute Myeloid Leukemia • Circular RNA • Circ-ANAPC7 • Microarray

\begin{abstract}
Background/Aims: Circular RNAs (circRNAs) are a family of novel non-coding RNAs associated with various diseases, especially cancer. Recent studies have demonstrated that circRNAs participate in pathogenesis mainly by acting as microRNA (miRNA) sponges. The expression profile of circRNAs in acute myeloid leukemia (AML) has rarely been reported. Methods: Profiles of circRNAs were analyzed using an Arraystar human circRNA microarray with 5 bone marrow samples from patients with newly diagnosed AML and 5 from patients with irondeficiency anemia. Quantitative reverse transcription PCR was used to validate the expression pattern of circRNAs. Furthermore, circRNA-miRNA network, Gene Ontology (GO), and Kyoto Encyclopedia of Genes and Genomes (KEGG) pathway analyses were applied. Results: CircRNA microarray analysis revealed that 698 circRNAs were differentially expressed in AML patients, with 282 circRNAs found to be upregulated and 416 to be downregulated. Quantitative reverse transcription PCR showed that circ-ANAPC7 was significantly upregulated in AML. Bioinformatics analysis predicted that circ-ANAPC7 acts as a sponge for the miR-181 family, KEGG analysis revealed that it is associated with cancer-related pathways, and GO analysis indicated that most of its target genes are involved in biological processes. Conclusions: These findings show that circ-ANAPC7 is a promising biomarker for AML, and that it might participate in AML pathogenesis by acting as a sponge for the miR-181 family.
\end{abstract}

Yun Yang, MD

and Aili $\mathrm{He}, \mathrm{MD}$
Department of Hematology, The Second Affiliated Hospital of Xi'an Jiaotong University

Xiwu Road 157, Xincheng District, Xi'an, Shaanxi 710004 (China)

Tel.+8615353699551, E-Mail yangyun108@163.com, heaili@xjtu.edu.cn 


\section{Cellular Physiology Cell Physiol Biochem 2018:47:1998-2007 \begin{tabular}{l|l} 
DOI: 10.1159/000491468 & and Biochemistry \\
Published online: July 03, 2018 & $\begin{array}{l}\text { 2018 The Author(s). Published by S. Karger AG, Basel } \\
\text { www.karger.com/cpb }\end{array}$
\end{tabular} Chen et al.: Expression Profiles of CircRNA in AML}

\section{Introduction}

Acute myeloid leukemia (AML) is a group of hematopoietic malignancies characterized by a poor prognosis. The pathogenesis of AML is closely associated with various genetic abnormalities that interfere with the growth, proliferation, and differentiation of normal hematopoietic progenitor cells $[1,2]$. These abnormalities often lead to rapidly occurring fatal infections, bleeding, or organ infiltration [3]. Overwhelming evidence has established a regulatory role for protein-coding genes in the tumorigenesis, maintenance, and progression of AML, while non-coding RNAs (ncRNAs), such as long non-coding RNAs (lncRNAs) and circular RNAs (circRNAs), have also been shown to be of great importance in AML [4].

CircRNAs are a family of novel ncRNAs that used to be identified as a by-product of abnormal RNA splicing with no biological function. More recently, accumulating evidence has shown that they play a crucial role in post-transcriptional regulation in vivo $[5,6]$. CircRNAs are characterized by a covalently closed loop structure lacking $3^{\prime}$ and $5^{\prime}$ ends. They are highly stable and mainly reside in the cytoplasm, and thus have the potential to be used as biomarkers [7]. A number of studies have found that circRNAs, such as CDas1R, have an important function in the pathogenesis of several diseases $[5,8,9]$. However, little is known about the role of circRNAs in human cancer.

CircRNAs have also been observed to act as microRNA (miRNA) sponges, which contribute to the regulation of gene expression. miRNAs are 23-nt ncRNAs that are implicated in numerous biological processes [10]. For instance, several studies have demonstrated that they function as guide RNAs leading to RNA silencing, and miRNA dysfunction is associated with the development of diseases such as leukemogenesis [11]. miRNAs also participate in post-transcriptional regulation in AML, leading to a cascade of downstream effects and indicating the potential for targeted therapeutic strategies. CircRNA-based therapy is a novel approach to modulate dysregulated miRNAs in AML. Hence, in this study, we investigated the circRNA-miRNA regulatory network in AML.

We first explored the expression profile of circRNAs in 5 AML samples and 5 irondeficiency anemia (IDA) samples (control group) that were collected from the bone marrow of patients. Then, we validated one circRNA (hsa_circRNA_101141) derived from the ANAPC7 gene locus, termed circANAPC7, which was frequently upregulated in patients with AML. Finally, we found that circANAPC7 may act as a miR-181 sponge by bioinformatics analysis.

\section{Materials and Methods}

\section{Patients and specimen collection}

This study involved 87 patients diagnosed with AML and 45 patients diagnosed with IDA as a control group. All individuals were of Han Chinese ethnicity and were recruited from the Second Affiliated Hospital of Xi'an Jiaotong University. None of the patients had previously undergone chemotherapy. All specimens were obtained from bone marrow aspiration, and after removal were immediately preserved at $-80^{\circ} \mathrm{C}$ until required. This study was approved by the Ethics Committee of the Second Affiliated Hospital of Xi'an Jiaotong University (No. 2015181) and met international standards for patient confidentiality. Written informed consent was obtained from all patients.

Total RNA extraction

Total tissue RNA was extracted from the AML and IDA samples using the TRIzol reagent (Invitrogen, Carlsbad, CA, USA) according to the manufacturer's instructions. The purity and concentration of RNA samples were determined with a NanoDrop ND-1000 (Thermo Fisher Scientific, Wilmington, DE, USA).

Microarray analysis

We selected five AML specimens and five IDA specimens for microarray analysis. Total RNA was treated with RNase R to enrich circular RNAs. The enriched circular RNAs were then amplified and transcribed into fluorescent cRNA utilizing random primers according to the Arraystar Super RNA Labeling protocol 


\section{Cellular Physiology Cell Physiol Biochem 2018:47:1998-2007 \begin{tabular}{l|l} 
DOI: 10.1159/000491468 & and Biochemistry \\
Published online: July 03, 2018 & $\begin{array}{l}\text { 2018 The Author(s). Published by S. Karger AG, Basel } \\
\text { www.karger.com/cpb }\end{array}$
\end{tabular} Chen et al.: Expression Profiles of CircRNA in AML}

(Arraystar, Inc., Rockville, MD, USA). The labeled circRNAs were hybridized onto an Arraystar Human circRNA Array $(8 \times 15 \mathrm{~K}$, Arraystar, Inc.), and then incubated at $65^{\circ} \mathrm{C}$ for $17 \mathrm{~h}$. After washing the array with gene

Table 1. qRT-PCR primer sequences

\begin{tabular}{lll}
\hline Primer name & Primer FW $\left(5^{\prime}-3^{\prime}\right)$ & Primer RV $\left(5^{\prime}-3^{\prime}\right)$ \\
\hline$\beta$-actin(H) & GTGGCCGAGGACTTTGATTG & CCTGTAACAACGCATCTCATATT \\
hsa_circRNA_101141 & GGGAGCAGCACTTAGGAACAT & AAAGCTGGTACTTCTGAGGTGG \\
hsa_circRNA_103164 & AGGACGAGCAGGGTGCAGAA & CTGACAGCGCAGTTTGAGGG \\
\hline
\end{tabular}
expression wash buffer (Agilent Technologies, Santa Clara, CA, USA), the slide was scanned with an Agilent Scanner G2505C.

CircRNA microarray data analysis

Data were extracted using Agilent Feature Extraction software. Data processing, including quantile normalization, was performed using R software. CircRNAs that were significantly differentially expressed between the AML and IDA groups were identified using a fold-change cut-off value or through volcano plot filtering. The prediction of miRNA targets for circRNAs and circRNA-miRNA interactions was conducted using the TargetScan and miRanda algorithms.

Validation of candidate circRNAs using quantitative reverse transcription polymerase chain reaction

Candidate circRNAs were selected for validation if they met the following criteria: 1) the raw intensity of all tested samples was greater than 500; 2) the P-value of the circRNAs between AML and IDA was $<0.01$; 3 ) the fold change in expression between AML and IDA was >2.5; and 4) the miRNAs in the predicted circRNA-miRNA-mRNA/IncRNA network has been proven to be associated with AML in previous studies. cDNA was synthesized by reverse transcription using a PrimeScript RT Master Mix with random primers according to manufacturer's protocols (TaKaRa Bio, Shiga, Japan). Then, quantitative reverse transcription PCR (qRT-PCR) was performed using SYBR Premix Ex Taq ${ }^{\mathrm{TM}}$ II (Tli RNaseH Plus) (TaKaRa) following the manufacturer's instructions. Divergent primers were synthesized by Invitrogen (Shanghai, China) and the sequences are shown in Table 1.

CircRNA-microRNA co-expression network

Validated candidate circRNAs were used as seeds to enrich a circRNA-miRNA-mRNA/IncRNA network according to the analysis of starBase v2.0 (http://starbase.sysu.edu.cn/) [12]. Cytoscape (http://www.cytoscape.org/) was applied to build a circRNA-miRNA-mRNA/lncRNA network. miRTarBase (http://mirtarbase.mbc.nctu.edu.tw/) was used to extract experimentally validated targets of the top 5 predicted miRNAs for validated candidate circRNAs [13].

Gene Ontology and Kyoto Encyclopedia of Genes and Genomes analyses

Kyoto Encyclopedia of Genes and Genomes (KEGG) enrichment analyses were carried out using the DAVID analysis tool (https://david.ncifcrf.gov/) to define further the biological pathways of the predicted miRNAs. Gene Ontology (GO) analysis was also performed to clarify the functional roles of miRNA target genes.

\section{Statistical analysis}

Results are reported as means \pm standard deviation for triplicate measurements. Significant differences between groups were estimated by the Mann-Whitney test using SPSS v.23.0 software (IBM, Chicago, IL, USA). $\mathrm{P}<0.05$ was considered statistically significant.

\section{Results}

Profiling of differentially expressed circRNAs in AML patients

The expression of 7441 human circRNAs was quantitated for the AML and IDA samples using a microarray platform. Hierarchical clustering and scatter plot visualization showed that circRNA expression levels were variable and distinguishable among the samples (Fig. 1).

\section{KARGER}


A total of 698 circRNAs were differentially expressed (fold change $\geq 2.0, \mathrm{P}<0.05$ ) between the AML and IDA samples, with 282 circRNAs found to be upregulated and 416 to be downregulated by more than 2-fold in AML patients compared with IDA patients. Analysis of the chromosomal distribution of circRNAs revealed that chromosome 1 had the greatest number of differentially expressed circRNAs, while mitochondrial DNA and chromosome 13 had the lowest number of differentially expressed circRNAs (Fig. 2a). The classification of all differentially expressed circRNAs is shown in Fig. 2b. Most differentially expressed circRNAs are transcribed from protein-coding exons, some are derived from introns, while a small number come from other sources. These data suggest that the expression of circRNAs in the bone marrow of AML patients differs from that in IDA patients.

Fig. 1. CircRNA microarray expression data. (A) Hierarchical clustering indicates differences in circRNA expression profiling between the AML and control groups. (B) A scatter plot was used to assess circRNA expression variation between the AML and control groups. The values of the $\mathrm{X}$ and $\mathrm{Y}$ axes in the scatter plot are the normalized signal values of the samples (log2 scaled). The middle green line indicates no difference between both groups. CircRNAs

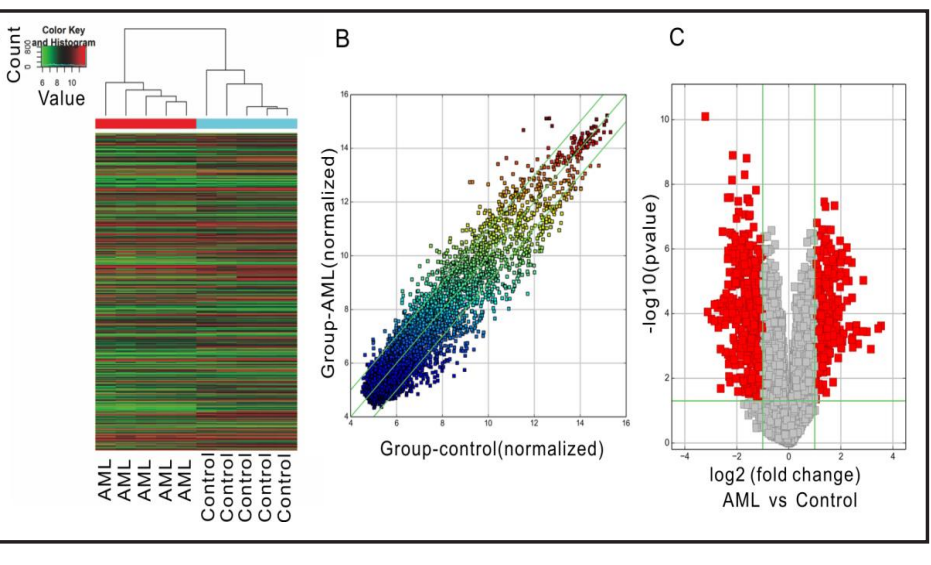
above the top green line or below the bottom green line indicate more than a 2-fold changes. (C) A volcano plot was applied to visualize expression profiling between both groups. The vertical green lines refer to a 2.0-fold ( $\log 2$ scaled) increase and decrease, respectively. The horizontal green line corresponds to a P-value of $0.05(-\log 10$ scaled). Each red point represents a differentially expressed circRNA with statistical significance.

Fig. 2. Distribution and category of differentially expressed circRNAs. (A) Distribution of differentially expressed circRNAs in human chromosomes. (B) CircRNA category is shown in the bar diagram. Most differentially expressed circRNAs originate from exons.

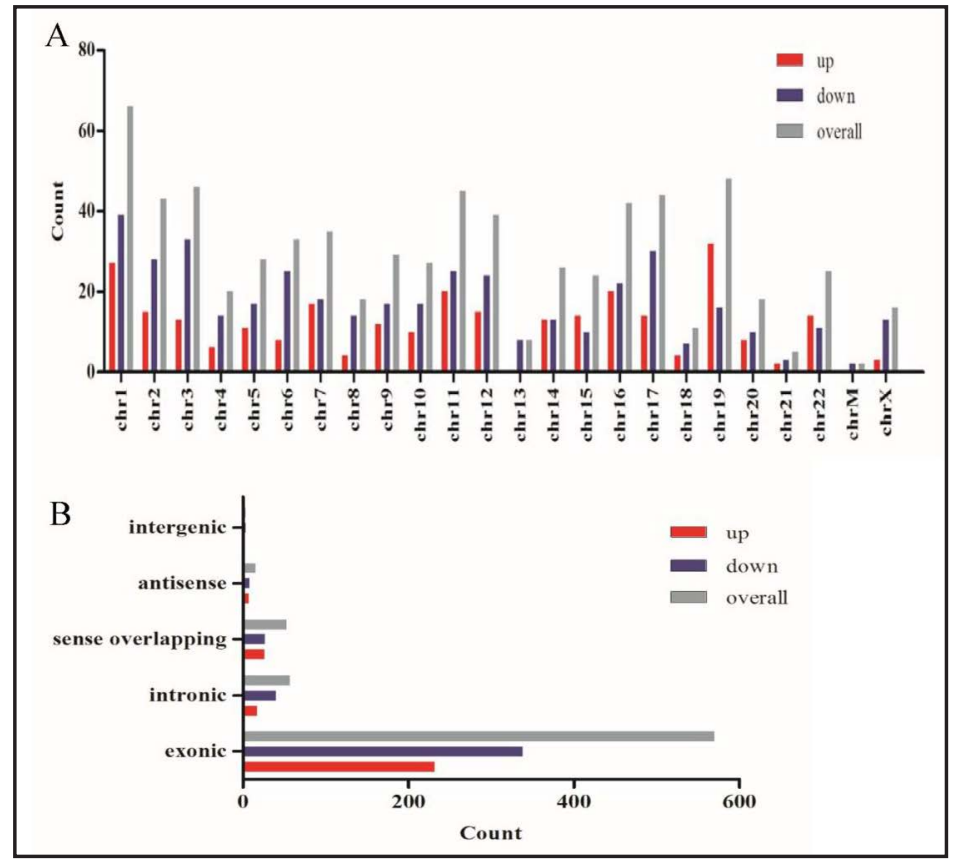


Fig. 3. Validation of circ-ANAPC7 expression by quantitative reverse transcription PCR. Circ-ANAPC7 is significantly upregulated in AML patients $(\mathrm{P}<0.001)$.

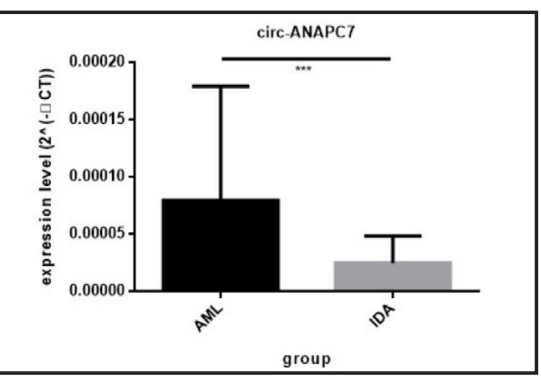

Fig. 4. CircRNA-miRNA-mRNA/lncRNA coexpression network. The red, yellow, purple, and blue nodes represent circRNA, miRNA, mRNA, and IncRNA, respectively.

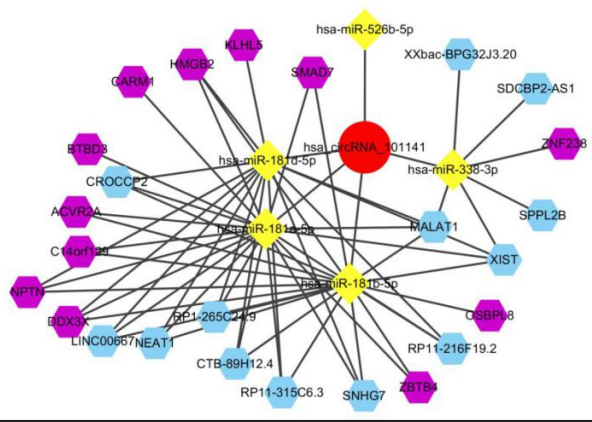

Fig. 5. Prediction of circRNA and miRNA interactions. The interactions of circ-ANAPC7 and the top 5 target miRNAs were predicted based on TargetScan and miRanda. The prediction for the interaction of circ-ANAPC7 with the miR181 family was completely consistent in both databases.

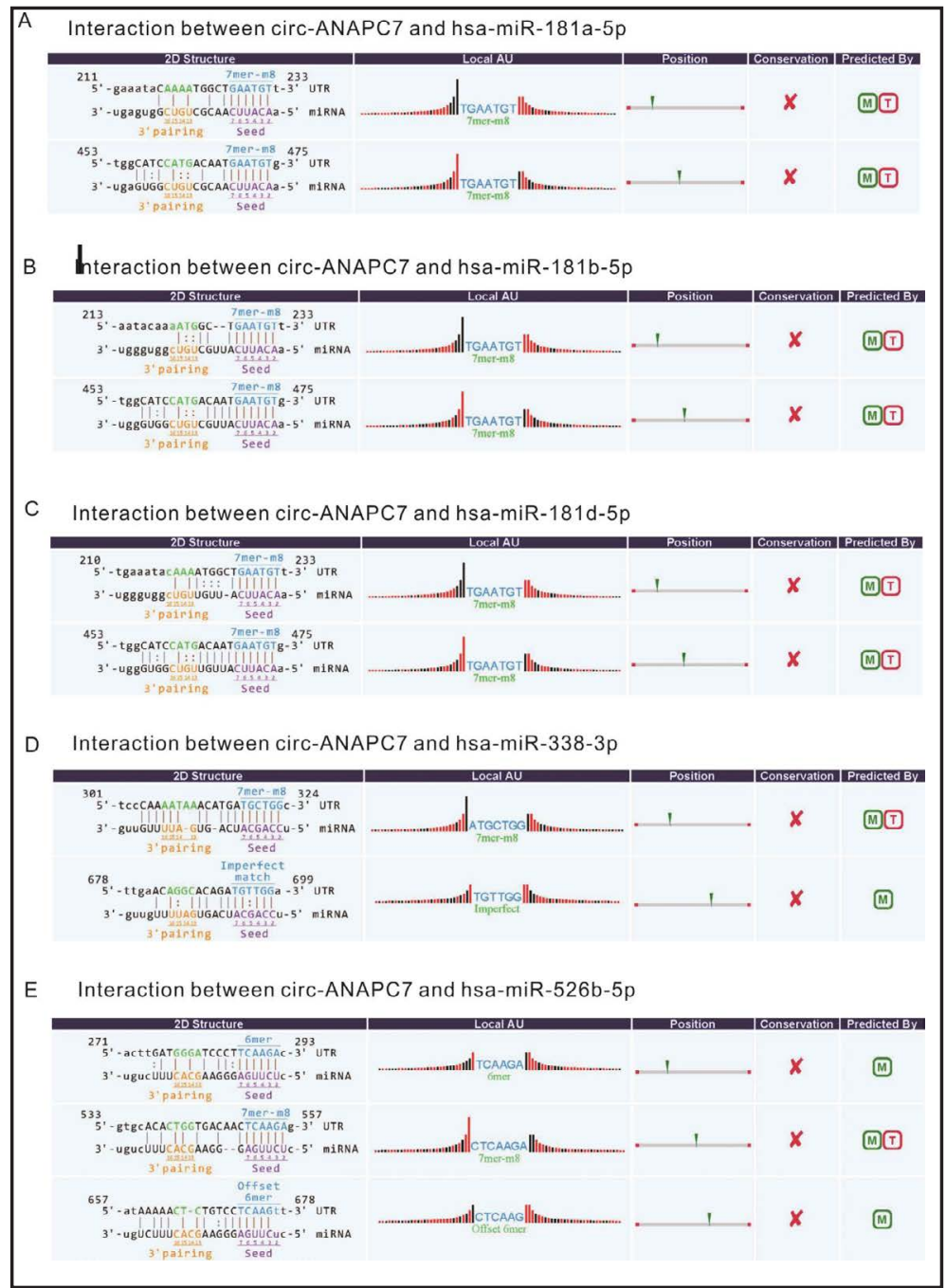


Table 2. Validated targets for circ-ANAPC7-related miRNAs

\begin{tabular}{|c|c|c|c|c|}
\hline miRNA & Reporter assay & Western blot & qRT-PCR & Total \\
\hline hsa-miR-181a-5p & $\begin{array}{l}\text { RGS5 RALA PRKCDATM TERT DDIT4 HIPK2 SIRT1 } \\
\text { ATG5 BCL2 WIF1 RAP1B E2F5 NLK GATA6 CDX2 } \\
\text { BCL2L11 PRAP1 FOS MTMR3 MCL1 XIAP GPR78 } \\
\text { PPP3CA TWIST1 RNF2 MAP2K1 ABCG2 MAPK1 } \\
\text { ZNF763 PROX1 KLF6 DDX3X KRAS PBX3 TIMP1 } \\
\text { PGR COL16A1 TGFBRAP1 TGFBR1 HRAS DUSP6 } \\
\text { PTPN11 DUSP5 PTPN22 NOTCH1 CDKN1B IFNG } \\
\text { KAT2B PLAG1 }\end{array}$ & $\begin{array}{l}\text { RGS5 RALA PRKCD ATM CD4 BCL2 WIF1 RAP1B } \\
\text { E2F5 RNF2 MAP2K1 ABCG2 MAPK1 ZNF763 } \\
\text { PROX1 BCL2L11 KLF6 DDX3X KRAS PBX3 } \\
\text { TIMP1 PGR COL16A1 TGFBRAP1 TGFBR1 HRAS } \\
\text { DUSP6 PTPN11 DUSP5 PTPN22 NOTCH1 } \\
\text { CDKN1B IFNG AHR STAT3 KAT2B PLAG1 }\end{array}$ & $\begin{array}{l}\text { RGS5 RALA PRKCD ATM CD4 TERT DDIT4 HIPK2 } \\
\text { SIRT1 ATG5 BCL2 WIF1 RAP1B E2F5 RNF2 MAP2K1 } \\
\text { ABCG2 MAPK1 PROX1 BCL2L11 KLF6 DDX3X KRAS } \\
\text { PBX3 TIMP1 PGR COL16A1 TGFBRAP1 TGFBR1 HRAS } \\
\text { CDKN1B C120RF29 PHACTR2 SASH1 PHLDA1 } \\
\text { MOSPD1 SRGAP1 UBL3 PHACTR4 EREG IFNG AHR } \\
\text { STAT3 }\end{array}$ & $\begin{array}{l}\text { RGS5 RALA PRKCD ATM TERT DDIT4 HIPK2 SIRT1 ATG5 BCL2 WIF1 } \\
\text { RAP1B E2F5 NLK GATA6 CDX2 BCL2L11 PRAP1 FOS MTMR3 MCL1 } \\
\text { XIAP GPR78PPP3CA TWIST1 RNF2 MAP2K1 ABCG2 MAPK1 ZNF763 } \\
\text { PROX1 KLF6 DDX3X KRAS PBX3 TIMP1 PGR COL16A1 TGFBRAP1 } \\
\text { TGFBR1 HRAS DUSP6 PTPN11 DUSP5 PTPN22 NOTCH1 CDKN1B } \\
\text { IFNG KAT2B PLAG1 CD4 AHR STAT3 C120RF29 PHACTR2 SASH1 } \\
\text { PHLDA1 MOSPD1 SRGAP1 UBL3 PHACTR4 EREG }\end{array}$ \\
\hline hsa-miR-181b-5p & $\begin{array}{l}\text { NLK GATA6 CDX2 TIMP3 PLAG1 GRIA2 VSNL1 } \\
\text { BCL2 KAT2B TCL1A CYLD RNF2 TMED7 SIRT1 } \\
\text { CBX7 XIAP MCL1 IGF1R E2F1 LATS2 PBX3 ATM } \\
\text { CREB1 RAP1B ADCY9 MAP2K1 FOS SIX2 }\end{array}$ & $\begin{array}{l}\text { TIMP3 PLAG1 BCL2 KAT2B TCL1A MAP3K10 } \\
\text { RNF2 TMED7 E2F1 LATS2 PBX3 ATM CREB1 } \\
\text { RAP1B ADCY9 FOS NFIA SIX2 }\end{array}$ & $\begin{array}{l}\text { TIMP3 BCL2 TCL1A MAP3K10 CYLD RNF2 TMED7 } \\
\text { LATS2 PBX3 CREB1 RAP1B ADCY9 FOS SIX2 }\end{array}$ & $\begin{array}{l}\text { NLK GATA6 CDX2 TIMP3 PLAG1 GRIA2 VSNL1 BCL2 KAT2B TCL1A } \\
\text { CYLD RNF2 TMED7 SIRT1 CBX7 XIAP MCL1 1GF1R E2F1 LATS2 PBX3 } \\
\text { ATM CREB1 RAP1B ADCY9 MAP2K1 FOS SIX2 MAP3K10 NFIA }\end{array}$ \\
\hline hsa-miR-181d-5p & BCL2 HRAS MGMT RAP1B & BCL2 HRAS MGMT RAP1B & BCL2 HRAS MGMT RAP1B & BCL2 HRAS MGMT RAP1B \\
\hline hsa-miR-338-3p & $\begin{array}{l}\text { PLA2G4B SMO CCND1 PREX2 SSX2IP NRP1 } \\
\text { MACC1 ZEB2 }\end{array}$ & $\begin{array}{l}\text { UBE2Q1 NOVA1 MAP1A DAB2IP ZNF238 SMO } \\
\text { MMP2 MMP9PREX2 SSX2IP NRP1 MACC1 ZEB2 }\end{array}$ & $\begin{array}{l}\text { UBE2Q1 NoVA1 MAP1A DAB2IP ZNF238 PLA2G4B } \\
\text { SMO MMP2 MMP9 PREX2 SSXZ2IP NRP1 MACC1 ZEB2 }\end{array}$ & $\begin{array}{l}\text { PLA2G4B SMO CCND1 PREX2 SSX2IP NRP1 MACC1 ZEB2 UBE2Q1 } \\
\text { NoVA1 MAP1A DAB2IP ZNF238 MMP2 MMP9 }\end{array}$ \\
\hline hsa-miR-526b-5p & none & none & none & none \\
\hline
\end{tabular}

Validation of differentially expressed CircRNAs by $q R T-P C R$

Considering the lower expression level of circRNAs and difficultly in overexpression functional test techniques, we selected only upregulated circRNAs for further validation according the criteria described in the Materials and Methods. Finally, circ-ANAPC7 (hsa_circRNA_101141) and circ-PI4KA (hsa_circRNA_103164) were tested by qRT-PCR in 87 AML and 45 IDA bone marrow samples. As shown in Fig. 3, circANAPC7 was significantly upregulated in AML patients, while circ-PI4KA showed a trend toward overexpression in AML patients, but this was not significant (data not shown). There was no significant difference in age and sex between the AML and IDA groups (data not shown).

\section{miRNA prediction and bioinformatics analyses}

Bioinformatics analysis showed that circ-ANAPC7 harbored miRNA response elements (MREs) for hsa-miR-181a-5p, hsa-miR-181b-5p, hsa-miR-181d-5p, hsa-miR-338-3p, and hsa-miR-526b$5 p$ (Fig. 4). This was confirmed by the starBase database for hsa-miR-181a-5p, hsa-miR-181b-5p, and hsa-miR-181d$5 p$ MREs, suggesting that circ-ANAPC7 might regulate miRNA-associated target genes in AML by acting as a sponge for the miR-181 family. To clarify the biological function of these five miRNAs, we extracted their validated targets from miRTarBase. miR-181a was shown to
Table 3. KEGG pathways

\begin{tabular}{|c|c|c|c|c|}
\hline KEGG pathway & $\mathrm{p}$-value & \#genes & \#miRNAs & Gene \\
\hline Pathways in cancer & 0.000995 & 11 & 4 & $\begin{array}{l}\text { WNT16 BCL2 } \\
\text { PDKN1B }\end{array}$ \\
\hline MAPK signaling pathway & 0.032874 & 8 & 3 & $\begin{array}{l}\text { PLA2G4B TAB2 KRAS BDNF GADD45G } \\
\text { NLK MAP2K1 PLA2G4C }\end{array}$ \\
\hline HTLV-I infection & 0.034562 & 8 & 2 & $\begin{array}{l}\text { WNT16 KRAS NFYB ATM NRP1 KAT2B } \\
\text { WNT3A WNT2 }\end{array}$ \\
\hline Long-term depression & $5.63 \mathrm{E}-08$ & 6 & 3 & $\begin{array}{l}\text { PLA2G4B GNAI3 KRAS GRIA2 MAP2K1 } \\
\text { PLA2G4C }\end{array}$ \\
\hline Melanogenesis & 0.001262 & 6 & 1 & $\begin{array}{l}\text { WNT16 GNAI3 KRAS WNT3A MAP2K1 } \\
\text { WNT2 }\end{array}$ \\
\hline Serotonergic synapse & 0.00542 & 6 & 2 & $\begin{array}{l}\text { PLA2G4B GNAI3 KRAS PTGS2 MAP2K1 } \\
\text { PLA2G4C }\end{array}$ \\
\hline VEGF signaling pathway & 0.000628 & 5 & 2 & PLA2G4B KRAS PTGS2 MAP2K1 PLA2G4C \\
\hline GnRH signaling pathway & 0.003231 & 5 & 2 & PLA2G4B KRAS MMP2 MAP2K1 PLA2G4C \\
\hline $\begin{array}{l}\text { Hedgehog signaling } \\
\text { pathway }\end{array}$ & 0.001553 & 4 & 2 & WNT16 WNT3A SMO WNT2 \\
\hline $\begin{array}{l}\text { Glycerophospholipid } \\
\text { metabolism }\end{array}$ & 0.003005 & 4 & 2 & PLA2G4B LCLAT1 LPGAT1 PLA2G4C \\
\hline Basal cell carcinoma & 0.003005 & 4 & 2 & WNT16 WNT3A SMO WNT2 \\
\hline $\begin{array}{l}\text { Fc epsilon RI signaling } \\
\text { pathway }\end{array}$ & 0.009002 & 4 & 2 & PLA2G4B KRAS MAP2K1 PLA2G4C \\
\hline Prostate cancer & 0.009311 & 4 & 3 & BCL2 CDKN1B KRAS MAP2K1 \\
\hline Thyroid cancer & 0.000609 & 3 & 1 & KRAS MAP2K1 CCDC6 \\
\hline $\begin{array}{l}\text { Dorso-ventral axis } \\
\text { formation }\end{array}$ & 0.000995 & 3 & 1 & NOTCH2 KRAS MAP2K1 \\
\hline Cocaine addiction & 0.009002 & 3 & 2 & GNAI3 BDNF GRIA2 \\
\hline Bladder cancer & 0.010895 & 3 & 2 & KRAS MMP2 MAP2K1 \\
\hline Notch signaling pathway & 0.014492 & 3 & 2 & NOTCH2 LFNG KAT2B \\
\hline
\end{tabular}

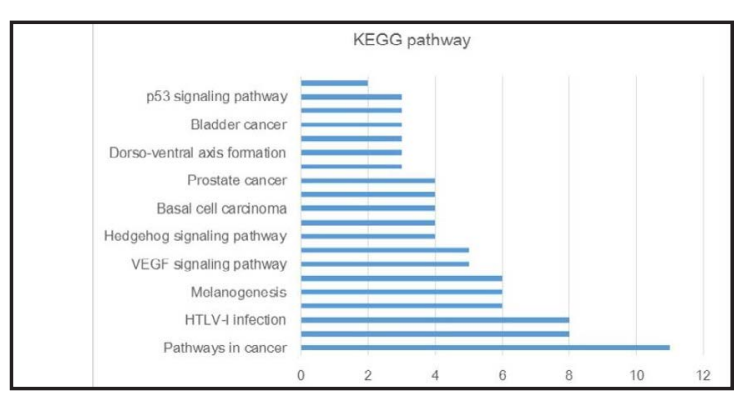

Fig. 6. KEGG pathway analysis. 


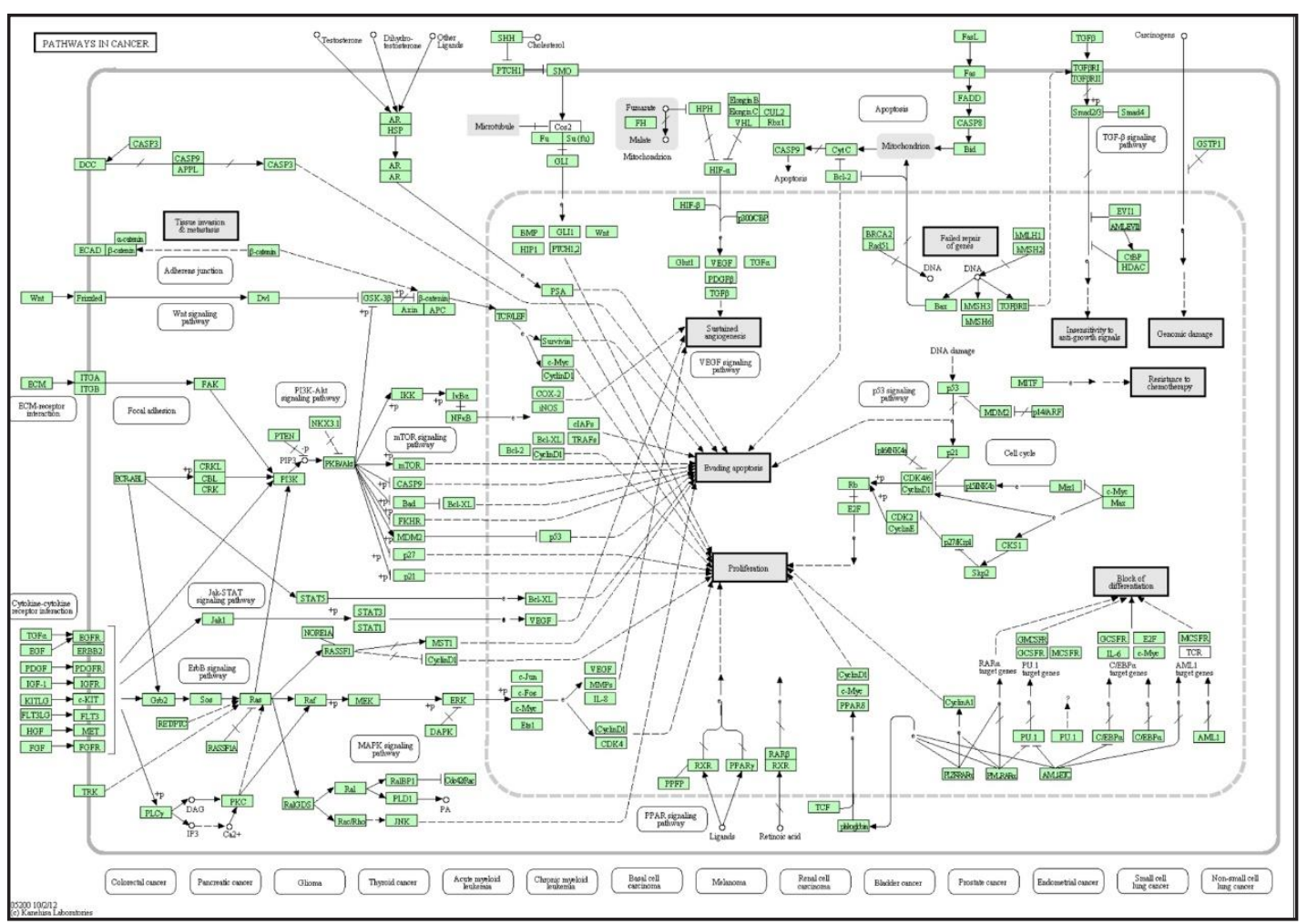

Fig. 7. Circ-ANAPC7 is related to pathways in cancer.

have the most targets, while miR-526 had none. The details of the validated targets of the five miRNAs are shown in Table 2.

To investigate further the molecular mechanism of circRNAs, the circ-ANAPC7miRNA-mRNA/lncRNA network was constructed by Cytoscape (Fig. 5). Moreover, because these miRNAs have been suggested to play important roles in oncogenesis, KEGG pathway enrichment analysis was conducted using DAVID (Fig. 6). The five miRNAs appeared to be strongly associated with cancer-related pathways (Fig. 7). Table 3 lists the combinatorial effect of the five miRNAs in the top 20 pathways and their target genes. GO enrichment analysis of circ-ANAPC7 target genes revealed that their properties could be defined as molecular function and cellular component (data not shown). These processes are closely associated with AML tumorigenesis.

\section{Discussion}

AML is a heterogeneous clonal malignancy characterized by the failure of hematopoietic proliferation [14]. Within China, the incidence of leukemia is approximately 4.44 per 100, 000 men and 3.09 per 100, 000 women [15]. Despite rapid advances in AML therapy, including the development of novel drug targets and transplantation, treatment has remained stagnant for the past few decades, with most patients relapsing and dying of complications [16]. Even with current treatment, the majority of patients older than 65 years will die of AML within 1 year $[17,18]$. To address this issue, research has focused on the identification of novel targets for AML therapy.

A wide range of evidence has indicated that ncRNAs such as circRNAs play an important role in leukemogenesis [19-21]; thus, circRNA-based therapy is a feasible approach for the treatment of AML. Aberrant circRNA expression has been associated with the malignant 
behavior of cancers $[22,23]$, but few studies have reported the potential roles of circRNAs in AML.

Here, we explored differences in the expression patterns of circRNAs between AML and IDA patients. Microarray analysis identified 282 circRNAs that were upregulated and 416 that were downregulated in AML, suggesting their potential involvement in disease pathogenesis. We further demonstrated by qRT-PCR that circ-ANAPC7 was significantly upregulated in AML, indicating that it could act as an oncogenic circRNA. Bioinformatics analysis predicted that circ-ANAPC7 functioned as a sponge for the miR-181 family because it harbored several MREs for this family. These results are consistent with our previous hypothesis that circRNAs are oncogenic in AML, and suggest that circ-ANAPC7 could be used as a biomarker for AML diagnosis or as a novel drug target.

The miR-181 family comprises miR-181a, miR-181b, miR-181c, and miR-181d, which are encoded by three different transcripts located on three chromosomes. All miR-181 family members contain similar seed sequences that differ by fewer than four nucleotides [24]. Previous studies have demonstrated that the miR-181 family regulates the differentiation of B cells, T cells, and natural killer cells during normal hematopoiesis [25-27]. miR-181a was reported to regulate non-small cell lung cancer proliferation and migration through targeting VCAM-1 [28]. In some tumors, the miR-181 family was shown to target the antiapoptotic genes BCL2 and MCL1, leading to apoptosis and the inhibition of tumor growth. Dysregulation of the miR-181 family was also shown to be closely associated with the pathogenesis and prognosis of AML. Additionally, members of the miR-181 family were demonstrated to mediate several cancer-related pathways, such as the RAS/MAPK pathway, thus acting as tumor suppressors in myeloid leukemogenesis [29, 30]. Therefore, targeting the circ-ANAPC7-miR-181 axis may be a novel therapeutic approach for AML.

This study has a number of limitations. First, we chose IDA patients as our control group because it is difficult to obtain bone marrow samples from healthy individuals. Second, we validated circ-ANAPC7 as a competitive endogenous RNA of the miR-181 family by only bioinformatics analysis, and not by experimental means. Finally, we did not elucidate the clinical significance of circ-ANAPC7 because of the short follow-up period. Further studies are therefore needed to determine the clinical significance and underlying mechanisms of circ-ANAPC7 in the pathogenesis of AML.

\section{Conclusion}

This study shows that circ-ANAPC7 is a promising biomarker for AML, and that it might participate in the pathogenesis of AML by acting as a sponge for the miR-181 family.

\section{Abbreviations}

miR (or miRNA; microRNA); circRNA; circular (RNA); ncRNA; non-coding (RNA); AML (acute myeloid leukemia); IDA; iron-deficiency (anemia); lncRNA; long (non-coding RNA); qRT-PCR; quantitative (reverse transcription PCR); GO; Gene (Ontology); KEGG; Kyoto (Encyclopedia of Genes and Genomes); MRE; miRNA (response element).

\section{Acknowledgements}

Yun Yang and Aili He contributed to the conception and design of this study and manuscript writing. Tian Liu, Jing Liu, and Ying Shen provided language assistance. Yuandong Feng, Xiaman Wang, Ju Bai, and Baiyan Wang helped to collect bone marrow samples from the patients. Wanhong Zhao, Juan Yang, and Yuqiang Ji assisted in proofreading this article. We thank all donors who donated to the microarray service at KangChen Biotech, Shanghai, 


\section{Cellular Physiology Cell Physiol Biochem 2018;47:1998-2007 \begin{tabular}{l|l} 
DOI: 10.1159/000491468 & O 2018 The Author(s). Published by S. Karger AG, Basel \\
www.karger.com/cpb
\end{tabular} \\ Chen et al.: Expression Profiles of CircRNA in AML}

China. This work was supported by Scientific and technological projects of Shaanxi province (2014-SF-010).

All procedures performed in studies involving human participants were in accordance with the ethical standards of the institutional and/or national research committee and with the 1964 Helsinki declaration and its later amendments or comparable ethical standards. Informed consent was obtained from all individual participants included in the study.

\section{Disclosure Statement}

No competing financial interests exist.

\section{References}

1 Schlenk RF, Dohner K, Krauter J, Frohling S, Corbacioglu A, Bullinger L, Habdank M, Spath D, Morgan M, Benner A, Schlegelberger B, Heil G, Ganser A, Dohner H: Mutations and treatment outcome in cytogenetically normal acute myeloid leukemia. N Engl J Med 2008;358:1909-1918.

2 Shih AH, Abdel-Wahab O, Patel JP, Levine RL: The role of mutations in epigenetic regulators in myeloid malignancies. Nat Rev Cancer 2012;12:599-612.

3 Estey E, Dohner H: Acute myeloid leukaemia. Lancet 2006;368:1894-1907.

4 Wei S, Wang K: Long noncoding RNAs: pivotal regulators in acute myeloid leukemia. Exp Hematol Oncol 2015;5:30.

5 Memczak S, Jens M, Elefsinioti A, Torti F, Krueger J, Rybak A, Maier L, Mackowiak SD, Gregersen LH, Munschauer M, Loewer A, Ziebold U, Landthaler M, Kocks C, le Noble F, Rajewsky N: Circular RNAs are a large class of animal RNAs with regulatory potency. Nature 2013;495:333-338.

6 Capel B, Swain A, Nicolis S, Hacker A, Walter M, Koopman P, Goodfellow P, Lovell-Badge R: Circular transcripts of the testis-determining gene Sry in adult mouse testis. Cell 1993;73:1019-1030.

7 Li Y, Zheng Q, Bao C, Li S, Guo W, Zhao J, Chen D, Gu J, He X, Huang S: Circular RNA is enriched and stable in exosomes: a promising biomarker for cancer diagnosis. Cell Res 2015;25:981-984.

-8 Barbagallo D, Condorelli A, Ragusa M, Salito L, Sammito M, Banelli B, Caltabiano R, Barbagallo G, Zappala A, Battaglia R, Cirnigliaro M, Lanzafame S, Vasquez E, Parenti R, Cicirata F, Di Pietro C, Romani M, Purrello M: Dysregulated miR-671-5p / CDR1-AS / CDR1 / VSNL1 axis is involved in glioblastoma multiforme. Oncotarget 2016;7:4746-4759.

-9 Hansen TB, Kjems J, Damgaard CK: Circular RNA and miR-7 in cancer. Cancer Res 2013;73:5609-5612.

10 Bartel DP: MicroRNAs: target recognition and regulatory functions. Cell 2009;136:215-233.

11 Liao Q Wang B, Li X, Jiang G: miRNAs in acute myeloid leukemia. Oncotarget 2017;8:3666-3682.

12 Li JH, Liu S, Zhou H, Qu LH, Yang JH: starBase v2.0: decoding miRNA-ceRNA, miRNA-ncRNA and proteinRNA interaction networks from large-scale CLIP-Seq data. Nucleic Acids Res 2014;42:D92-97.

13 Chou CH, Chang NW, Shrestha S, Hsu SD, Lin YL, Lee WH, Yang CD, Hong HC, Wei TY, Tu SJ, Tsai TR, Ho SY, Jian TY, Wu HY, Chen PR, Lin NC, Huang HT, Yang TL, Pai CY, Tai CS, Chen WL, Huang CY, Liu CC, Weng SL, Liao KW, Hsu WL, Huang HD: miRTarBase 2016: updates to the experimentally validated miRNA-target interactions database. Nucleic Acids Res 2016;44:D239-247.

14 De Kouchkovsky I, Abdul-Hay M: 'Acute myeloid leukemia: a comprehensive review and 2016 update'. Blood Cancer J 2016;6:e441.

15 Chen W, Zheng R, Baade PD, Zhang S, Zeng H, Bray F, Jemal A, Yu XQ He J: Cancer statistics in China, 2015 CA Cancer J Clin 2016;66:115-132.

16 Saultz JN, Garzon R: Acute Myeloid Leukemia: A Concise Review. J Clin Med 2016;5:

17 Meyers J, Yu Y, Kaye JA, Davis KL: Medicare fee-for-service enrollees with primary acute myeloid leukemia: an analysis of treatment patterns, survival, and healthcare resource utilization and costs. Appl Health Econ Health Policy 2013;11:275-286.

18 Ofran Y, Tallman MS, Rowe JM: How I treat acute myeloid leukemia presenting with preexisting comorbidities. Blood 2016;128:488-496. 


\section{Cellular Physiology Cell Physiol Biochem 2018;47:1998-2007 \begin{tabular}{l|l} 
DOI: 10.1159/000491468 & O 2018 The Author(s). Published by S. Karger AG, Basel \\
www.karger.com/cpb
\end{tabular} \\ Chen et al.: Expression Profiles of CircRNA in AML}

19 Xing CY, Hu XQ, Xie FY, Yu ZJ, Li HY, Bin Z, Wu JB, Tang LY, Gao SM: Long non-coding RNA HOTAIR modulates c-KIT expression through sponging miR-193a in acute myeloid leukemia. FEBS Lett 2015;589:1981-1987.

20 Hao S, Shao Z: HOTAIR is upregulated in acute myeloid leukemia and that indicates a poor prognosis. Int J Clin Exp Pathol 2015;8:7223-7228.

-21 Wang YX, Zhang TJ, Yang DQ, Yao DM, Yang L, Zhou JD, Deng ZQ, Ma JC, Guo H, Wen XM, Lin J, Qian J: Reduced miR-215 expression predicts poor prognosis in patients with acute myeloid leukemia. Jpn J Clin Oncol 2016;46:350-356.

-22 Zheng Q, Bao C, Guo W, Li S, Chen J, Chen B, Luo Y, Lyu D, Li Y, Shi G, Liang L, Gu J, He X, Huang S: Circular RNA profiling reveals an abundant circHIPK3 that regulates cell growth by sponging multiple miRNAs. Nat Commun 2016;7:11215.

23 Guarnerio J, Bezzi M, Jeong JC, Paffenholz SV, Berry K, Naldini MM, Lo-Coco F, Tay Y, Beck AH, Pandolfi PP: Oncogenic Role of Fusion-circRNAs Derived from Cancer-Associated Chromosomal Translocations. Cell 2016;166:1055-1056.

-24 Sun X, Sit A, Feinberg MW: Role of miR-181 family in regulating vascular inflammation and immunity. Trends Cardiovasc Med 2014;24:105-112.

25 Chen CZ, Li L, Lodish HF, Bartel DP: MicroRNAs modulate hematopoietic lineage differentiation. Science 2004;303:83-86.

26 Garzon R, Croce CM: MicroRNAs in normal and malignant hematopoiesis. Curr Opin Hematol 2008;15:352358.

27 Li X, Zhang J, Gao L, McClellan S, Finan MA, Butler TW, Owen LB, Piazza GA, Xi Y: MiR-181 mediates cell differentiation by interrupting the Lin28 and let-7 feedback circuit. Cell Death Differ 2012;19:378-386.

28 Cao Y, Zhao D, Li P, Wang L, Qiao B, Qin X, Li L, Wang Y: MicroRNA-181a-5p Impedes IL-17-Induced Nonsmall Cell Lung Cancer Proliferation and Migration through Targeting VCAM-1. Cell Physiol Biochem 2017;42:346-356.

29 Huang X, Schwind S, Santhanam R, Eisfeld AK, Chiang CL, Lankenau M, Yu B, Hoellerbauer P, Jin Y, Tarighat SS, Khalife J, Walker A, Perrotti D, Bloomfield CD, Wang H, Lee RJ, Lee LJ, Marcucci G: Targeting the RAS/ MAPK pathway with miR-181a in acute myeloid leukemia. Oncotarget 2016;7:59273-59286.

30 Weng H, Lal K, Yang FF, Chen J: The pathological role and prognostic impact of miR-181 in acute myeloid leukemia. Cancer Genet 2015;208:225-229. 